\title{
UCRL-CONF-205549
}

LAWRENCE LIVERMORE NATIONAL LABORATORY

\section{Fielding of an Imaging VISAR Diagnostic at the National Ignition Facility (NIF)}

R.M. Malone, J.R. Bower, G.A. Capelle, J.R.

Celeste, P.M. Celliers, B.C. Frogget, R.L. Guyton, M.I. Kaufman, G.A. Lare, T.L. Lee, B.J. MacGowan, S. Montelongo, E.W. Ng, T.L. Thomas, T.W. Tunnell, P.W. Watts

July 2004

SPIE International Symposium Optical Science and Technology $49^{\text {th }}$ Annual Meeting, Denver, Colorado, August 2-6, 2004 
This document was prepared as an account of work sponsored by an agency of the United States Government. Neither the United States Government nor the University of California nor any of their employees, makes any warranty, express or implied, or assumes any legal liability or responsibility for the accuracy, completeness, or usefulness of any information, apparatus, product, or process disclosed, or represents that its use would not infringe privately owned rights. Reference herein to any specific commercial product, process, or service by trade name, trademark, manufacturer, or otherwise, does not necessarily constitute or imply its endorsement, recommendation, or favoring by the United States Government or the University of California. The views and opinions of authors expressed herein do not necessarily state or reflect those of the United States Government or the University of California, and shall not be used for advertising or product endorsement purposes. 


\title{
Fielding of an imaging VISAR diagnostic at the National Ignition Facility (NIF)
}

\author{
Robert M. Malone ${ }^{*}$, John R. Bower ${ }^{\mathrm{b}}$, Gene A. Capelle ${ }^{\mathrm{c}}$, John R. Celeste ${ }^{\mathrm{b}}$, Peter M. Celliers ${ }^{\mathrm{b}}$, \\ Brent C. Frogget ${ }^{a}$, Robert L. Guyton ${ }^{d}$, Morris I. Kaufman ${ }^{a}$, Gregory A. Lare ${ }^{a}$, Tony L. Lee ${ }^{b}$, \\ Brian J. MacGowan ${ }^{b}$, Sam Montelongo ${ }^{b}$, Edmund W. Ng ${ }^{b}$, Thayne L. Thomas ${ }^{b}$, Thomas W. \\ Tunnell $^{\text {a }}$, Phillip W. Watts ${ }^{\mathrm{d}}$,

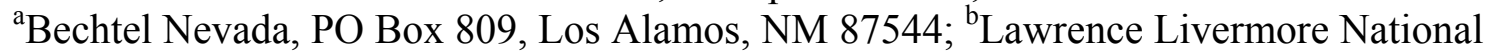 \\ Laboratory, PO Box 808, Livermore, CA 94551; ${ }^{\mathrm{c} B e c h t e l ~ N e v a d a, ~} 5540$ Ekwill St., Santa

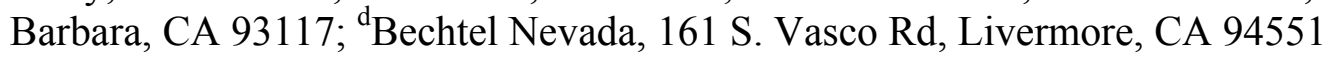

\begin{abstract}
The National Ignition Facility (NIF) requires diagnostics to analyze high-energy density physics experiments. As a core NIF early light diagnostic, this system measures shock velocities, shock breakout times, and shock emission of targets with sizes from 1 to $5 \mathrm{~mm}$. A $659.5 \mathrm{~nm}$ VISAR probe laser illuminates the target. An 8-inch-diameter fused silica triplet lens collects light at $\mathrm{f} / 3$ inside the 33 -foot-diameter vacuum chamber. The optical relay sends the image out an equatorial port, through a 2-inch-thick vacuum window, and into two VISAR (Velocity Interferometer System for Any Reflector) interferometers. Both streak cameras and CCD cameras record the images. Total track is 75 feet. The front end of the optical relay can be temporarily removed from the equatorial port, allowing for other experimenters to use that port. The first triplet can be no closer than $500 \mathrm{~mm}$ from the target chamber center and is protected from debris by a blast window that is replaced after every event. Along with special coatings on the mirrors, cutoff filters reject the NIF drive laser wavelengths and pass a band of wavelengths for VISAR, for passive shock breakout light, or for thermal imaging light (bypassing the interferometers). Finite Element Analysis was performed on all mounting structures. All optical lenses are on kinematic mounts, so that the pointing accuracy of the optical axis can be checked. A two-color laser alignment scheme is discussed.
\end{abstract}

Keywords: VISAR, optical relay, interferometer, National Ignition Facility (NIF), streak camera

\section{INTRODUCTION}

$\operatorname{VISAR}^{1,2}$ (Velocity Interferometry System for Any Reflector) measures the velocity of a moving surface by recording its Doppler wavelength shift. An optical imaging system has been designed to collect images from inside a 33-foot-diameter vacuum chamber at NIF. ${ }^{3}$ Fig. 1 shows four (quad) drive laser beam lines entering into the NIF target chamber and focused onto a sample target. VISAR makes use of one of the equatorial ports of this vacuum chamber. The Diagnostic Instrumentation Manipulator (DIM) used by VISAR has been designed for multiple users. The VISAR DIM cart hardware was designed to be compatible with existing DIM hardware designs.

DOE/NV/11718--881. This work was supported by the U. S. Department of Energy, National Nuclear Security Administration Nevada Site Office, under Contract No. DE-AC08-96NV11718.

*malonerm@nv.doe.gov; phone 1505 663-2014; fax $1505663-2003$.

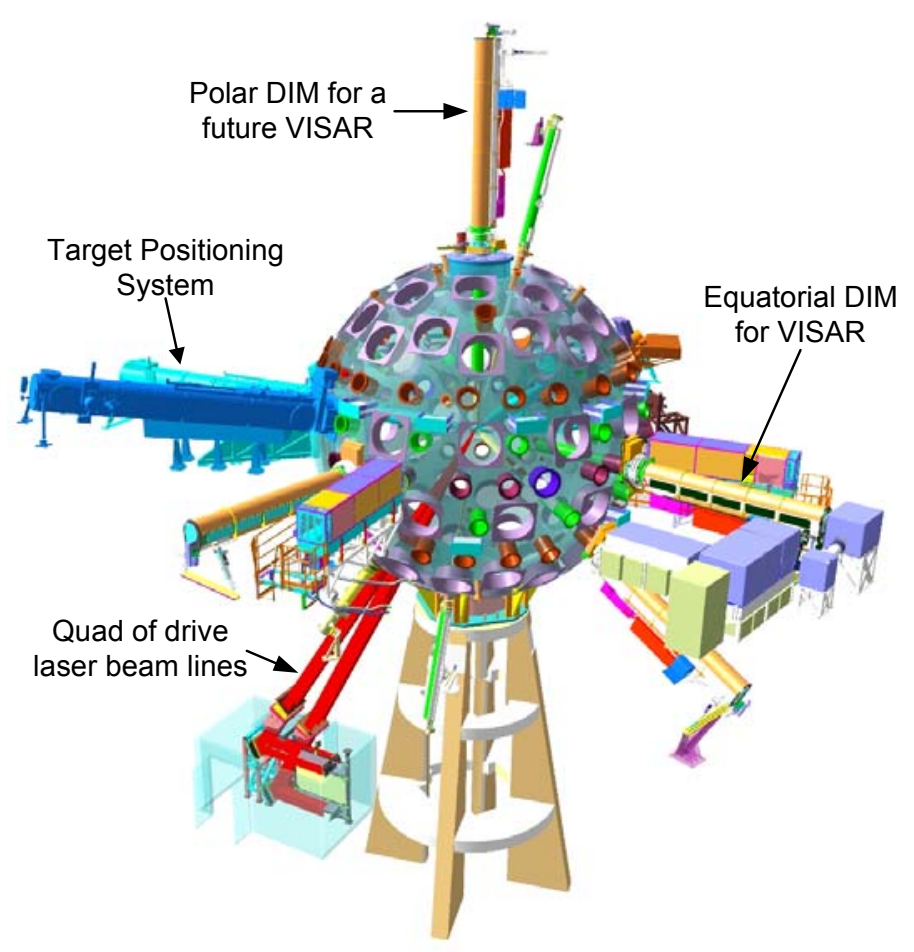

Fig. 1. NIF target chamber. 


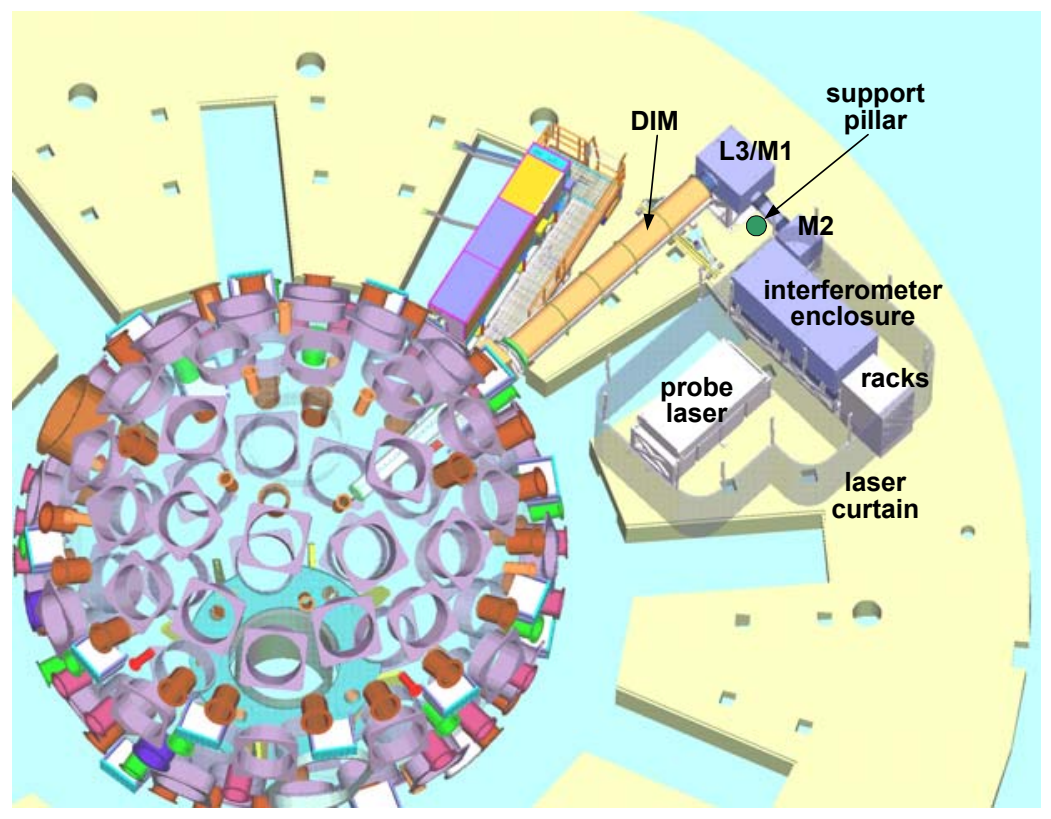

Fig. 2. VISAR work area at 90,45 port.

Fig. 2 shows the layout for this imaging VISAR, which routes the optical axis around a facility support pillar. A protective laser curtain encloses this diagnostic. Optics and mounting hardware inside the DIM are removable. The L3/M1 and M2 boxes, pinned to the concrete floor, are detachable so that other diagnostics may make use of this DIM location. The interferometer and laser tables are stationary. All enclosures for the DIM vacuum gate valve, DIM vacuum window, L3/M1, $\mathrm{M} 2$, interferometer table, and the probe laser table have safety interlock switches.

The DIM uses motor-controlled bipod legs for beam steering. Mirror M1 is oversized to allow for this steering; however, doublet lens L3 must be shifted to find the correct optical axis.

M1 has two tilting actuators under remote control. L3 has two tilt plus two translation actuators under remote control. M2 is 20 inches lower in elevation than M1. M2 has two remote tilting actuators and is used to set the optical beam height for the interferometer table.

\section{OPTICAL DESIGN}

The optical design has to be flexible to allow for changes in the placement of mirror boxes and the interferometer table. Because of the 500-mm stay out zone at the Target Chamber Center (TCC) and the desire to collect light at $\mathrm{f} / 3$, the first fused silica triplet lens is 8 inches in diameter. Diffraction-limited resolution (down to a few microns) for 1- to 5-mm objects is achieved at a distance of 75 feet.

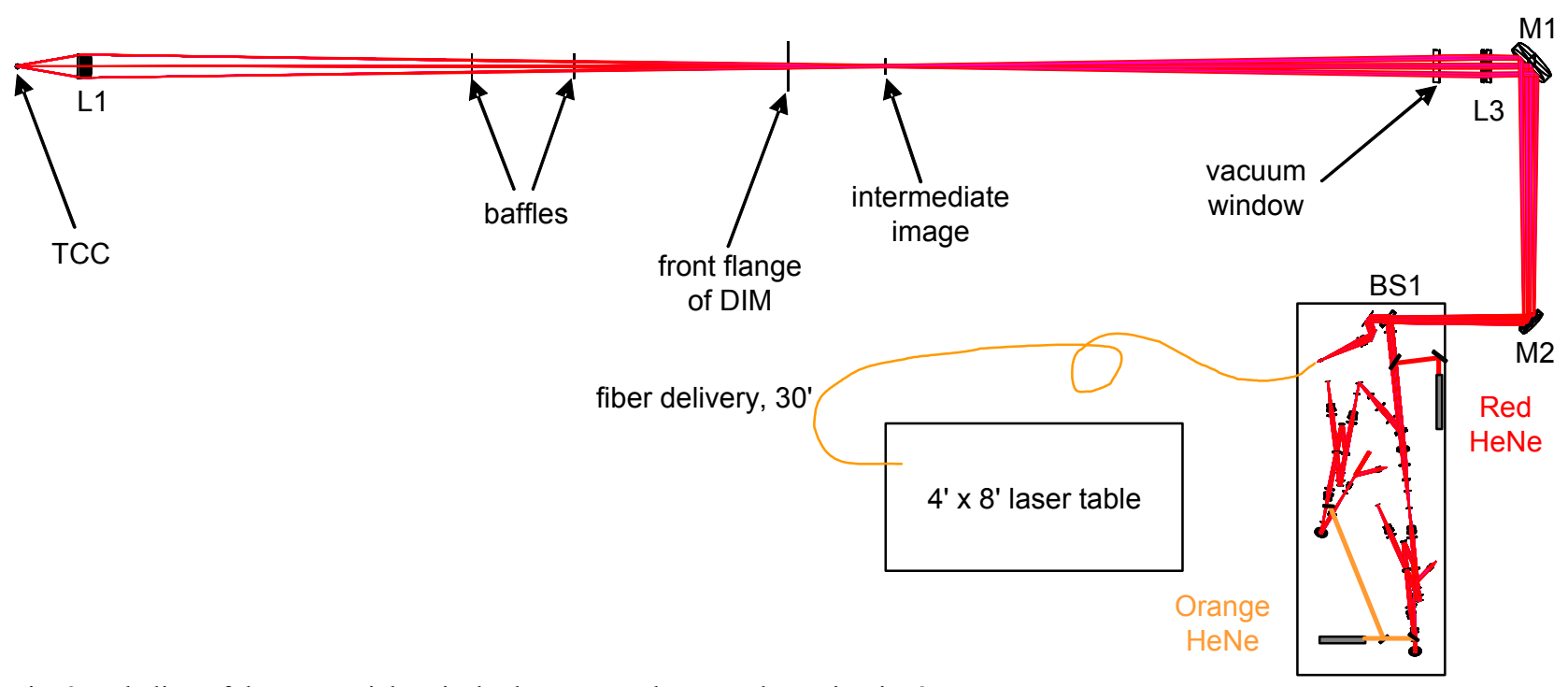

Fig. 3. Labeling of the equatorial optical relay system that was shown in Fig. 2. 
The light from the TCC is collected by a triplet lens (L1) and exits the DIM through a 2-inch-thick vacuum window (see Fig. 3). An intermediate image is formed inside the DIM and is picked up by a doublet lens (L3) that is placed in front of the first turning mirror (M1). A second turning mirror (M2) lowers the optical path to the interferometer table.

Light from a $60-\mathrm{kW}$ probe laser (see section 6) is delivered by three 1-mm optical fibers. This light enters the optical system through a 50/50 beam splitter, BS1. Half of the probe laser power is lost to a carbon beam dump, which prevents unshifted probe laser light from entering into the interferometer. Light from the TCC also makes use of this beam splitter. Half of the VISAR return signal is also lost at this beam splitter.

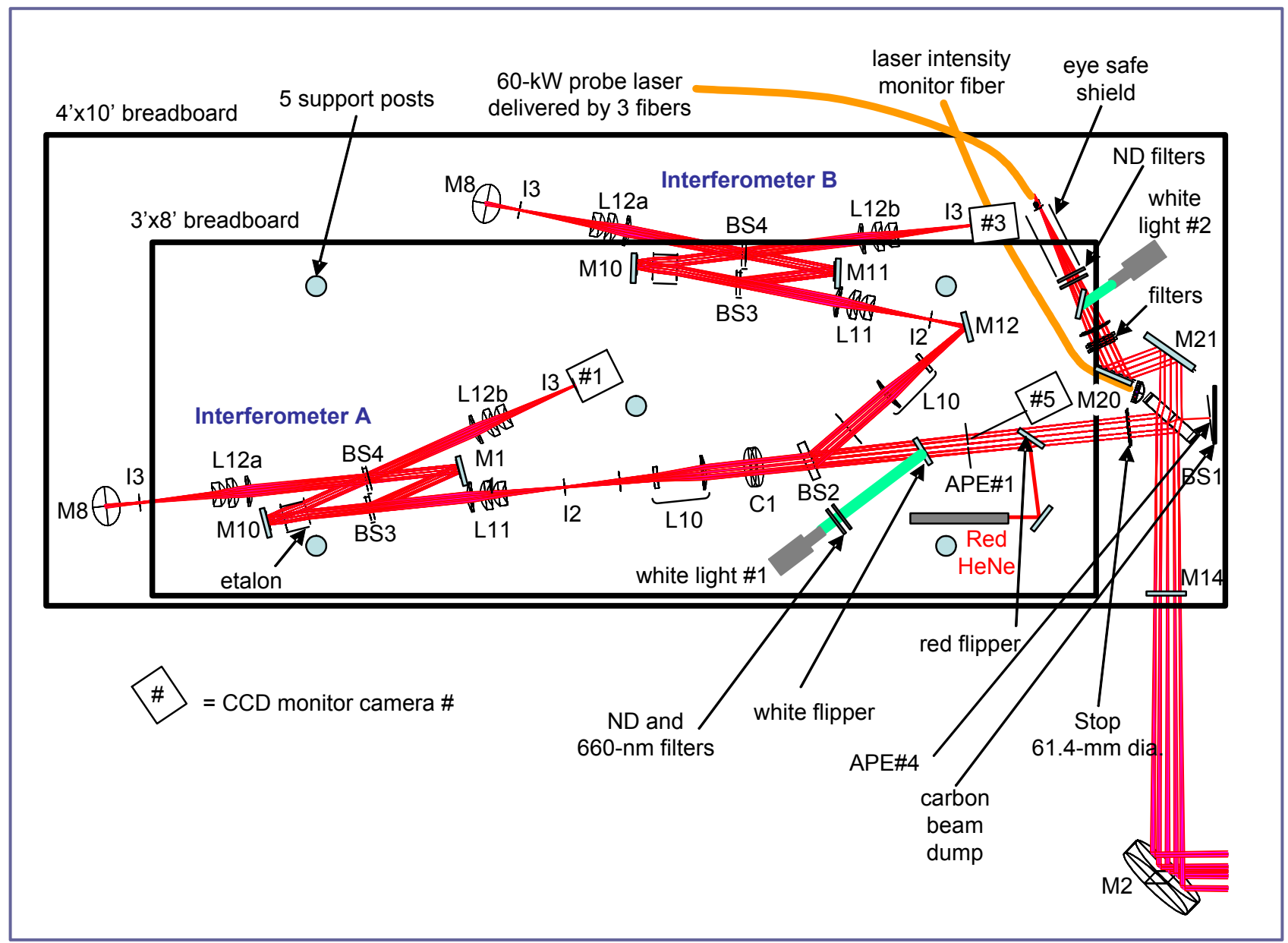

Fig. 4. Optical ray trace through lower level of interferometer table.

Because of space constraints, two interferometers are located on the lower shelf of the interferometer table, as shown in Fig. 4, while the recording streak cameras are located on the upper level of the table (Fig. 5). At the interferometer table, beam splitter BS2 splits the light into two separate interferometers. Each interferometer can have its own etalon (different velocity range) and its own image rotation, using a 37- $\mathrm{mm}$ aperture dove prism. A 1-1 optical relay is placed after the interferometer (image plane I3) and relays the image from the lower to the upper breadboard level (image plane I4). The dove prism is placed in the waist of the collimated section of this 1-1 optical relay.

Fig. 4 illustrates the entrance of a red HeNe alignment laser into the optical system by way of a flip mirror. This laser is centered on the slit of the streak camera and also on the output coupler of an orange HeNe alignment laser (Fig. 5). The orange HeNe laser enters the optical system also using a flip mirror and is centered on the output coupler of the red HeNe alignment laser as well as aperture cards APE\#1 and APE \#4 (Fig. 4). APE\#1 and APE\#4 are used to maintain 
the orange laser axis that ultimately terminates at the TCC. APE\#4 is permanently mounted. APE\#1, mounted on a kinematic plate, is removed when images are being recorded by the streak cameras. A paper reticule, with circles ranging from 5 to $25 \mathrm{~mm}$, is mounted on the back side of APE\#1; in this way CAM5 can view the back side of APE\#1 and record the tilt misalignment of the target placed at the TCC.

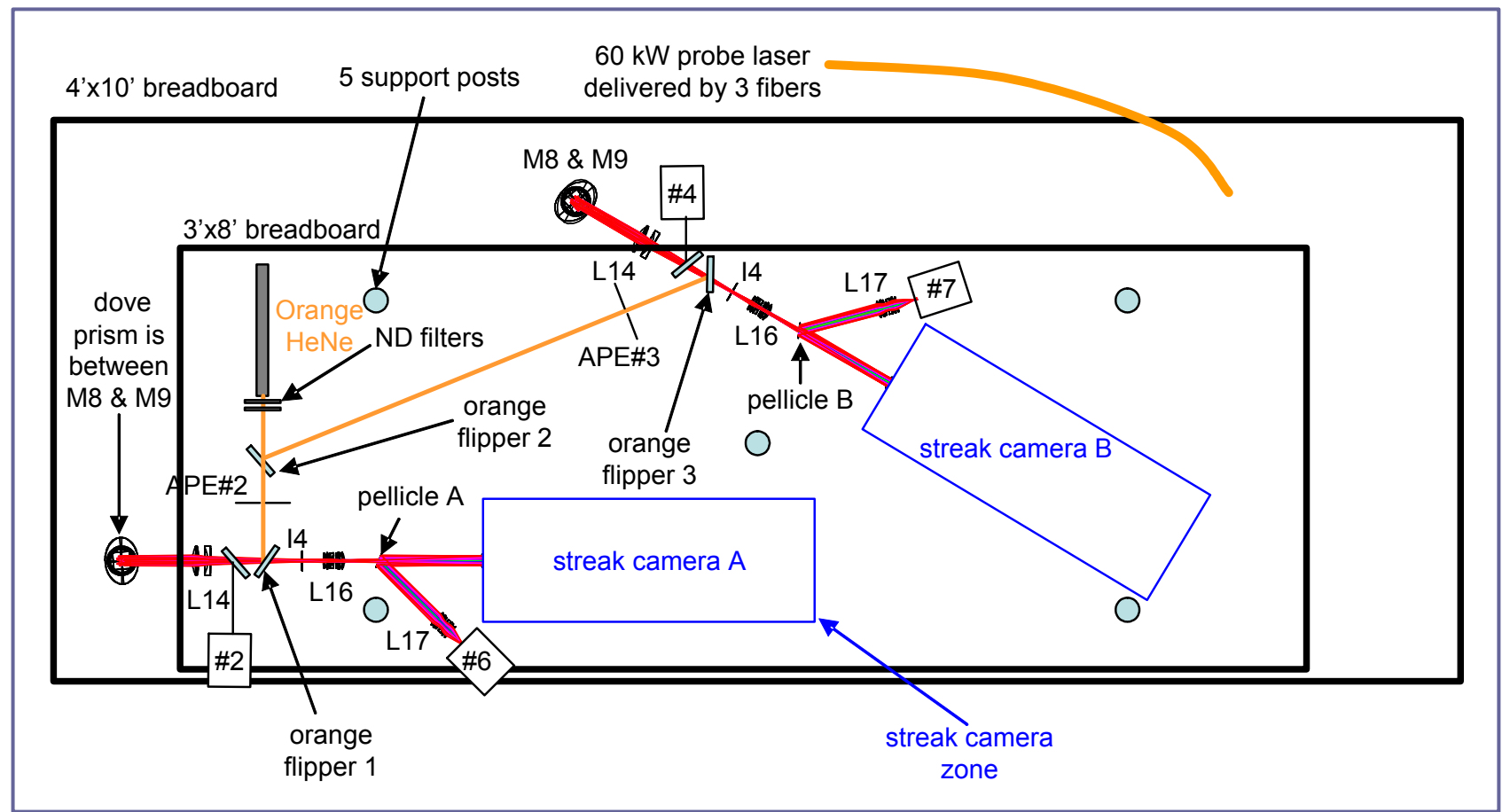

Fig. 5. Optical ray tracing through upper level of interferometer table. Using orange flipper 2, the orange laser is sent either through interferometer $\mathrm{A}$ or $\mathrm{B}$.

To minimize damage to expensive optical components, mirrors M1 and M2 have special coatings that do not reflect 1053-nm, 527-nm, or the 351-nm drive laser wavelengths. All optical elements, including mirrors, from the TCC to beam splitter BS1 (on the interferometer table) are fused silica to minimize radiation darkening caused by the intense $\mathrm{x}$ rays of some NIF experiments. Narrowband filters $(3 \mathrm{~nm})$ and cutoff filters are placed behind dove prisms where the relayed light is almost collimated. This provides additional protection to the streak camera data from the NIF drive lasers. There is also a wider 70-nm filter in the laser launch section to prevent NIF drive lasers from focusing onto the fiber end tip.

The original optical design used pairs of Nikkor lenses placed between image plane I4 and the slit of the streak camera. The Nikkor lenses were mated together with a flange that secured these lenses into a dual-angle tilt mount. By changing the ratio of focal lengths of the two Nikkor lenses, different magnification could be applied to the image presented to the slit of the streak camera. This is how we could record object sizes ranging from 1 to $5 \mathrm{~mm}$ in diameter. However, the focusing and aligning of this Nikkor pair proved to be time-consuming. In addition, we wished to reduce the number of optical elements in front of this slit. The Nikkor lenses have general broadband AR coatings. At 660-nm, internal reflection losses would reduce the contrast of the fringes.

The solution was to use a single 50-mm Nikkor lens (L16) to relay the image from I4 to the slit of the streak camera. The streak camera was mounted onto ball bearing guide rails and could easily be positioned. However, depending on the exact position of the $50-\mathrm{mm}$ lens, the magnification could not be calibrated. As a solution, a 50/50 pellicle was mounted onto a kinematic plate and placed between the 50-mm lens and the slit. Light from this pellicle is picked up by a macro lens and viewed by a CCD camera (note CAM6 and CAM7 positions in Fig. 5). For purposes of optical layout - to direct all the field points through macro lens L17 and into CAM6 and CAM7-a curved mirror (620-mm focal length) was temporally substituted at the streak camera slit plane (Fig. 5). Thus, this curved mirror acts like a field 
lens. The slit was anodized black and diffuse enough to allow equal reflection of all field points into CAM6 and CAM7. Consequently, when using CAM6 and CAM7 with the white light \#1, we are able to view the focusing of the I2 reticules. White light \#1 or \#2 are introduced into the optical system using flipper mirrors. Images of the magnifications are archived using a frame grabber. When the 660-nm CW light is used, CAM6 and CAM7 allow us to set the fringes perpendicular to the slits. Before collecting data with the probe laser, the I2 reticules and the pellicles are removed.

The comb and FIDU trigger optical fibers are glued into a 90-degree bent stainless steel tube and mounted on both sides of the streak camera slit. The available slit width for the line image is $19 \mathrm{~mm}$.

Image plane I2 is assigned as the reference plane. If the DIM is inaccessible, either white light \#1 or the $660-\mathrm{nm} \mathrm{CW}$ laser can reflect off a removable mirror M14 (Fig. 4). Either light source provides images to the monitoring CCD cameras. Most of the optical alignment takes place without access to the DIM. When access to the target at TCC is available, L1 is used to focus and overlap the object at the established I2 plane. Lens L10 can also be used for focus. For fine focus of the target at the TCC, white light \#2 can also be used; this eliminates the speckle image produced by laser illumination of the target.

\section{2-INCH LENS SURROGATE SYSTEM}

Before a new diagnostic can be delivered to NIF, it must pass off-line acceptance testing. Since this was a complicated optical imaging system, the alignment system had to be demonstrated before installation could be authorized by NIF. Streak camera recording and probe laser operations had to be demonstrated. The lead time for delivery of optical elements and mirrors was long. To manage risk and meet schedule, a surrogate imaging system was designed that employed 2-inch diameter lenses borrowed from a lens kit. ${ }^{3}$

Because of the long optical path length, optical elements were laid out onto three optical tables in two optical labs. The probe laser was located in a third lab and its light fed into a 1-mm-diameter optical fiber. The surrogate system was limited to relaying 1- to 3-mm-sized objects with f/14 light collection efficiency. Alignment procedures were demonstrated and acceptance testing was successful. Fig. 6 shows an example of a section of the optical relay that used substitute lenses. Focal lengths of lenses were chosen by using the two-lens formula:

$$
\frac{1}{f_{\mathrm{efl}}}=\frac{1}{f_{1}}+\frac{1}{f_{2}}-\frac{d}{f_{1} * f_{2}} .
$$

An optical lens design program was used to fine-tune the air spacings. All pairs of surrogate lenses were mounted onto translation stages, which allowed all lenses to be moved out of the optical axis so that the two color laser alignment scheme (see next section) could be evaluated. For the first two months of VISAR installation at NIF, while waiting for delivery of the large optics, the 2-inch-lens surrogate system was used.

\section{ALIGNMENT SYSTEM}

Maintaining stability over a very long optical path length was challenging. The shear number of optical elements made alignment complex. Alignment checks have to be done on $>60$ optical components (some having seven glass elements). Compound angles exist. Numerous VISAR operators are expected to use this diagnostic with different setups (etalon thickness, magnifications).

In order to establish the optical axis, all optical lens groups are mounted on kinematic plates. After clearing all lenses from the optical axis, each lens group is individually tested for centering and tilt. By using two differently colored lasers propagating in opposite directions, very exact alignment can be achieved. The optical elements have a V-coat for 660 $\mathrm{nm}$, and provide reasonable reflections at 612- and 633-nm wavelengths. Fig. 7 demonstrates that an operator using only one laser beam could correct a lens decenter by tilting it. This would be insufficient alignment because a tilt can compensate a decenter (Fig. 7D). This would not give the correct alignment as can be seen when using the two counterpropagating laser beams (Fig. 7E). 


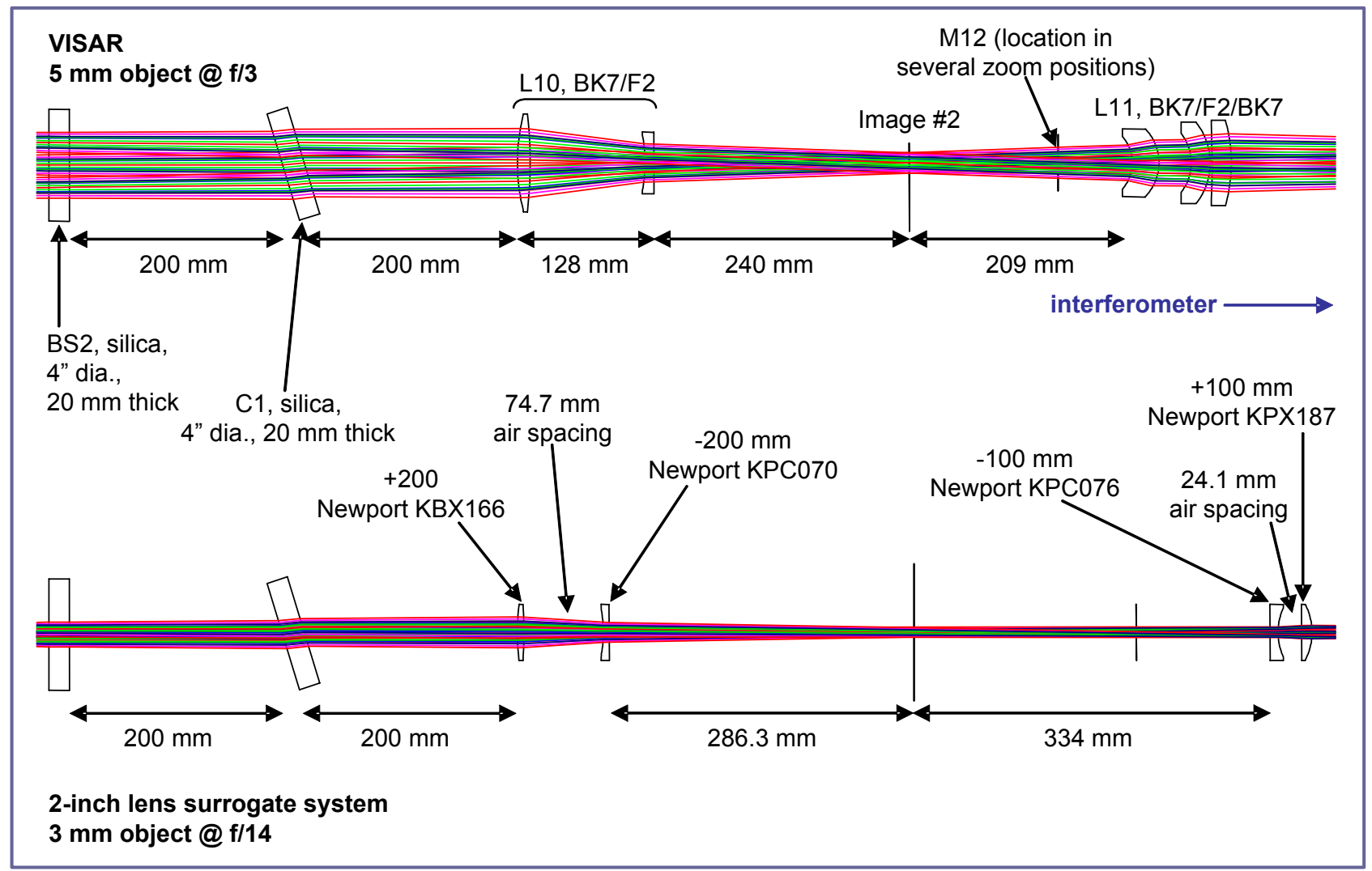

Fig. 6. 2-inch lens surrogate system replaces the $\mathrm{f} / 3$ system during early fielding and alignment operations.

Using a telescope or an autocollimator for alignment usually limits the viewing of alignment errors to the operator. These alignment aids would have to repeatedly be repositioned to cover the optics located on both levels inside the interferometer enclosure. By using aperture cards to display the two-colored laser alignment errors lets multiple users sample/probe for possible adjustments. Operators have found this method uncomplicated.

\section{OPTOMECHANICAL ASSEMBLY}

The mechanical designs have to be earthquake tolerant. Excluding the laser system there are over 60 optical components. One component weighs 49 pounds (M1). Weight of the L1 housing with its glass is 20 pounds. One component has 7 optical elements $(50 \mathrm{~mm} \mathrm{f} / 1.2$ Nikkor lens). The total thickness of glass and mirrors is almost 3 feet.

We designed and fabricated mounting gimbals for the L3 lens doublet, the M1 mirror, and the M2 mirror. The

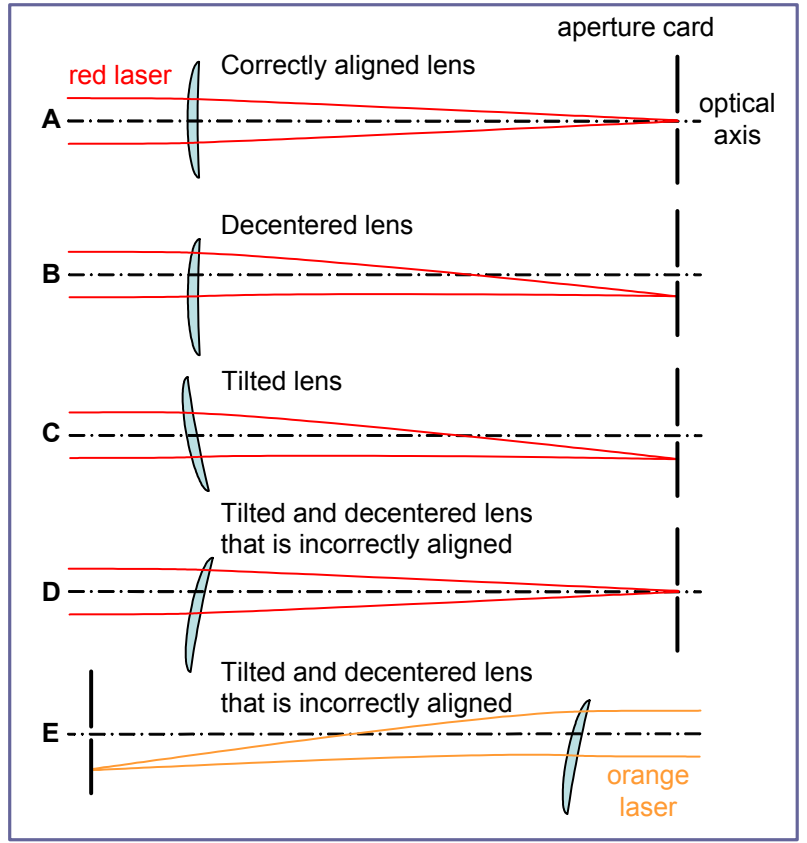

Fig. 7. How tilt and decenter of a lens can be used to recenter an image spot. 
optical elements were nonstandard sizes and no commercial mounts would satisfy design constraints. The L3 doublet lens (10.5-inches) has to move 10 inches to clear the optical axis during target alignment operations. M1 and M2 mirror boxes have to be removed during installation of any other DIM carts that use this equatorial port.

The DIM has a 2-stage extension to deliver an instrument close to the TCC. All diagnostics use a standard extension tube with a precision traveling screw. Each target chamber diagnostic requires a unique package that travels inside this extension tube for placement. In Fig. 8 the VISAR diagnostic is fully extended past the vacuum gate valve towards the TCC. Because of the 2-stage extension method used to get L1 close to the TCC, it was difficult to mount other relay lenses inside the DIM. Therefore, the next relay lens (L3) is located just outside the large 10.5-inch-diameter vacuum port window (seen in Fig. 9). Cross hairs are mounted onto the L1 frame as an orange laser alignment aid.

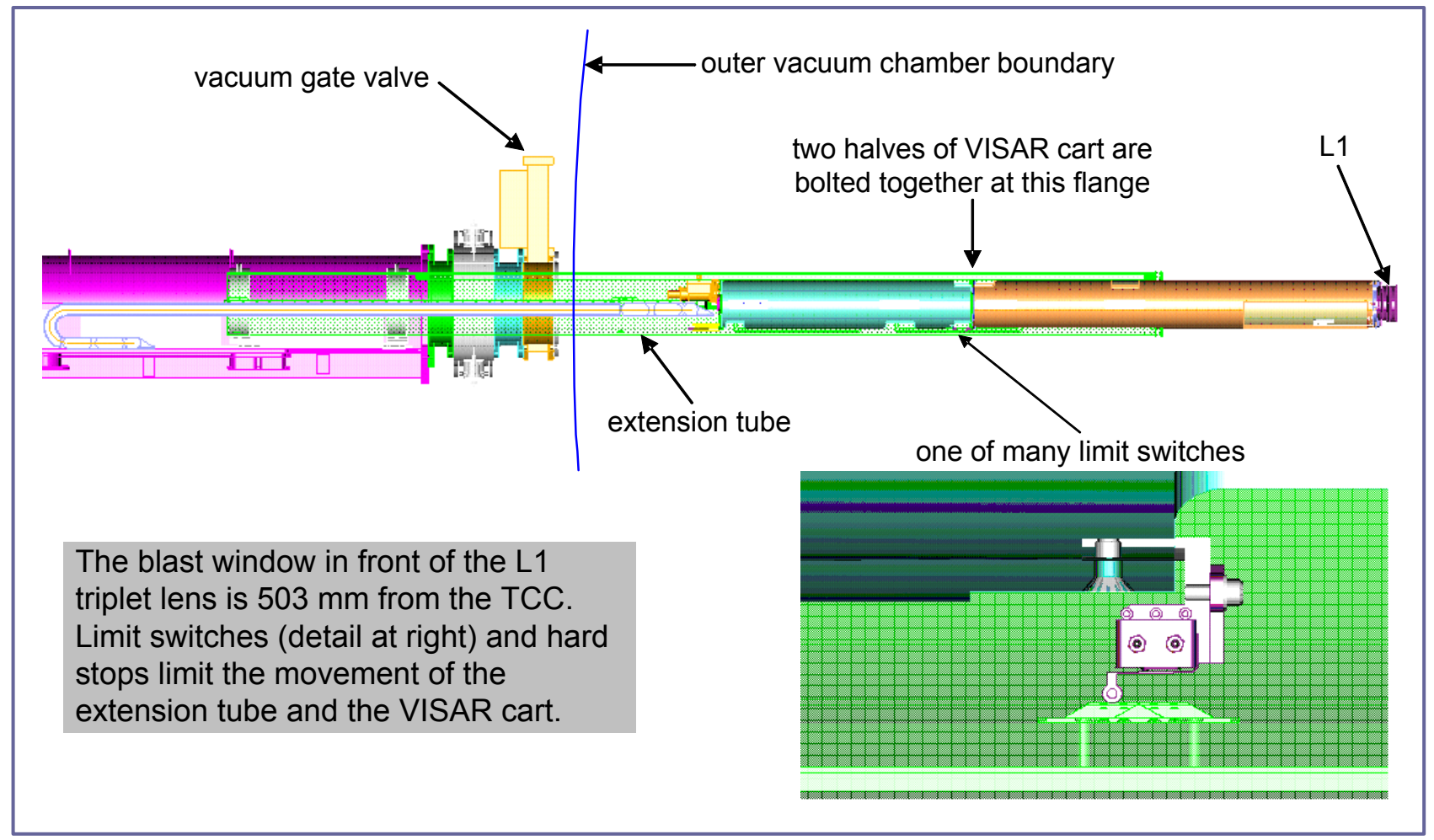

Fig. 8. VISAR Diagnostic cart fully extended.

The VISAR diagnostic cart that is inserted into the DIM is a 2-section design. The L1 triplet lens is mounted to the front of this DIM cart. Limit switches and hard stops guarantee that the VISAR cart will not overshoot and fall into the vacuum chamber. Fine focus of L1 is achieved by driving the precision extension drive screw.

Fig. 9 pictures the VISAR cart retracted outside the vacuum chamber and ready for servicing. An earlier design had a cart length of 17.5 feet with difficult access for removal of the L1 lens. To improve the design, a field lens (L2) placed close to a baffle at the cart motor mounting plate was eliminated and the diameters of the vacuum window, L3 relay doublet lens, and the M1 mirror were increased. After repositioning the cart baffles, no vignetting or loss of resolution resulted from these changes. Now the L1 doublet lens and its blast window have easy access through a side panel of the DIM. The blast window may have to be replaced after every event.

To make the assembly of the L1 triplet lens easier, we slotted its spacer rings. The housing was non-anodized aluminum. The spacer rings were stainless steel. By slotting the rings, we were able to seat the lenses without added friction force and this also prevented metal abrasion dust from entering the inside of the housing. The blast window has its own housing which mates to the front of the L1 housing. Two screws, in a push-pull arrangement, secure the removable blast window to the $\mathrm{L} 1$ housing. 


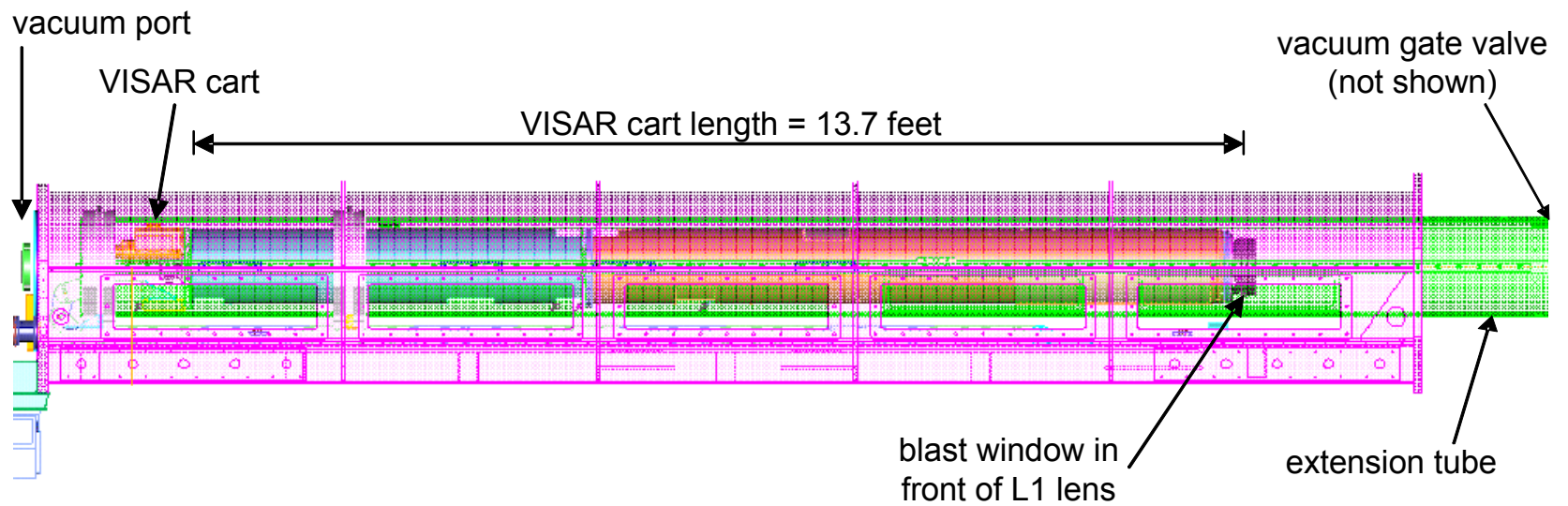

Fig. 9. Diagnostic cart in retracted position. Blast window can be swapped out through the vacuum port.

There are numerous motors, shutters, flippers, and magnetic switches that are controlled by a diagnostic computer. Position settings are archived. Configuration management requires all component changes to be updated into a spread sheet.

Fig. 10 is a model of one of two transport carriers used to insert the VISAR diagnostic into the DIM. This transport carrier was used during VISAR cart fabrication to fix problems prior to shipment to NIF, and it has the same guide rails and limit switches as the DIM. We were able to pre-tilt the L1 triplet lens to cancel calculated DIM sag for a fully inserted system by using this carrier. This gave us confidence that we could deliver a finished product the first time. All new diagnostic efforts at NIF are using this transport carrier design as a means to help designers manage their manufacturing risk, preventing lost time if DIM fit problems occur that would require extra machining.

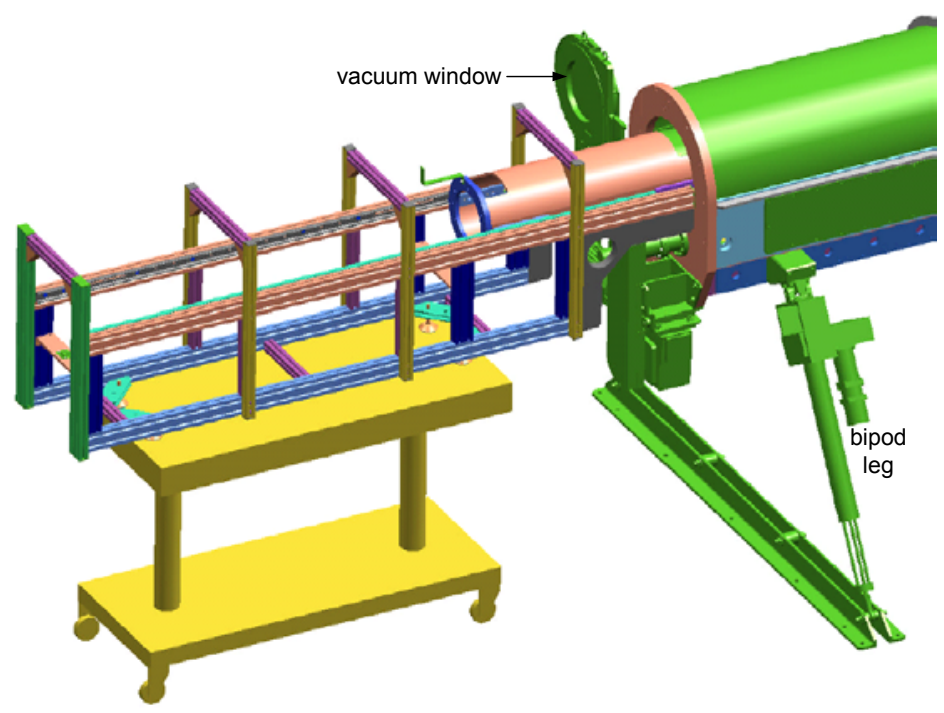

Fig. 10. Two cart carrier transporters (one for the front half and one for the back half) are used to prealign limit switches and set the tilts of L1 lens prior to insertion into the DIM.

\section{VISAR PROBE LASER SYSTEM}

The $60-\mathrm{kW}$ probe laser used in this VISAR system is currently one of the world's most powerful doubled Nd:YAG lasers operating at $659.5 \mathrm{~nm}$ and was obtained from Continuum Laser Corporation. ${ }^{4}$ The probe laser wavelength had to be far enough away from the three NIF drive laser wavelengths (Fig. 11) and it had to have a narrow line width (single frequency) for VISAR operations. This laser was chosen to lase at $1319 \mathrm{~nm}$ starting with a CW seed. If the $\mathrm{Nd}$ :YAG laser amplifiers are driven too hard, $1064 \mathrm{~nm}$ is difficult to suppress. The gain of the amplifiers had to be reduced and the number of laser amplifiers had to be increased from 7 to 14. Laser rods had to be double AR coated to keep the $1064 \mathrm{~nm}$ wavelength from becoming parasitic. The laser is optimized at a sub-Hertz repetition rate so that it will stay aligned when fired only occasionally. 


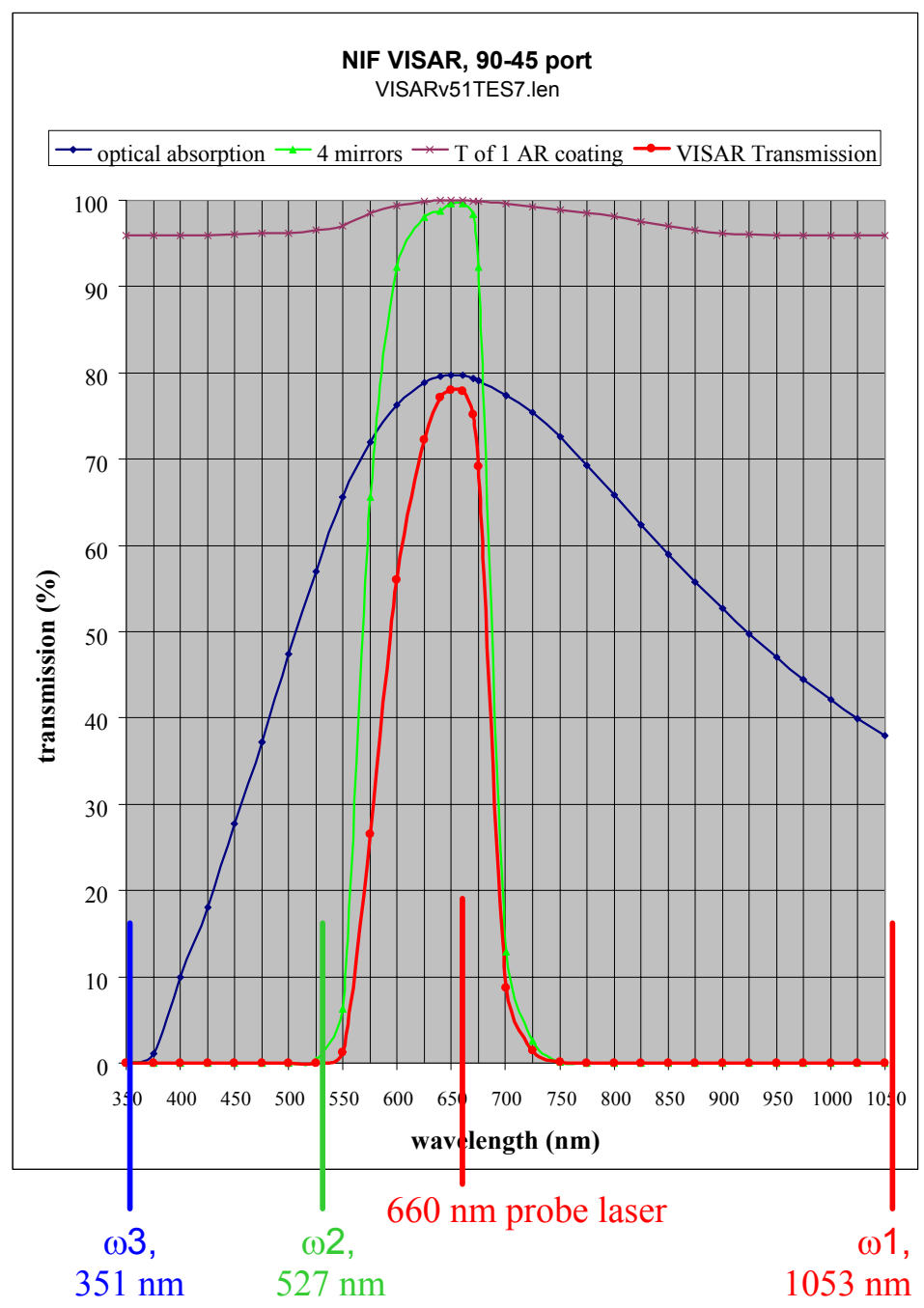

Fig. 11. Transmission curves for VISAR imaging. The $660-\mathrm{nm}$ probe laser is positioned away from NIF drive laser wavelengths.

It was discovered that another low gain laser line at $1340 \mathrm{~nm}$ had to be suppressed with better selective coatings on the turning mirrors between the laser amplifiers. Initial polarizers used thick film coatings that were hygroscopic. As the laser heated up, their reflectance changed. Thin film polarizers had to be received from a different manufacturer. The final laser design has a very long beam path and tends to drift with temperature changes. We intend to install heaters in the circulation water to reduce warm up time from four hours to minutes. We will maintain the laser enclosure environment at the elevated operation temperature. The heated water will require installing a UV sterilizer to prevent algae buildup.

There are three delivery fibers that transmit laser light to the interferometer table. Three fibers were chosen to minimize the risk of Brillouin scattering that could occur if too much laser light is injected. A cube beam splitter system splits the laser light into three jumper fibers that have 1-mm core diameters and are 1-meter long. An energy meter is used to equalize the light while adjusting half-wave plates. Then, the light is coupled into the delivery fibers that are 30 feet long. Using a flip mirror inside the laser table enclosure, either the $660-\mathrm{nm} \mathrm{CW}$ diode laser or the pulsed probe laser is allowed to enter into the delivery fibers. The $660-\mathrm{nm} \mathrm{CW}$ diode laser has variable power up to $200 \mathrm{~mW}$.

\section{SUMMARY}

Unraveling these VISAR diagnostic problems demanded innovative problem solving in lens design, optical alignment, laser design, mechanical design, system integration, and project management. Our plan was to insert a 2-inch lens surrogate optical system at NIF before the large optics arrived. Designing the surrogate optical system enabled the accelerated field schedule to run smoothly. The proper optics were used when installation was complete. The VISAR transport carrier designed for the DIM-based diagnostic is now required of all new NIF diagnostics.

We were able to maintain diffraction limited optical performance for the imaging. For a 5$\mathrm{mm}$ field of view, 1 part in 700 can be resolved by the VISAR imaging. For a 1-mm field of view, 1 part in 250 has been resolved.

We designed the ability to quickly remove all optics from beam path for alignment checks. The two-colored laser alignment scheme is accurate and compatible with optical systems that have a large number of components. The flexible optical relay system can accommodate a future thermal imaging system that is under development. Highspeed framing camera recording of images can be accommodated in this design.

\section{ACKNOWLEDGEMENTS}

The authors would like to thank the following Bechtel Nevada personnel for optomechanical support: Jim Daniel, Kevin McGillivray, Steve Slavin, Bill Skarda, and Aric Tibbitts. Peter Celliers of LLNL is the principal VISAR diagnostic physicist. $\mathrm{Ed} \mathrm{Ng}$ of LLNL is the VISAR project manager. Brian MacGowan is the NIF target diagnostic manager. 
Copyright Statement. By acceptance of this article, the publisher and/or recipient acknowledges the U.S. Government's right to retain a nonexclusive, royalty-free license in and to any copyright covering this paper.

Disclaimer. This report was prepared as an account of work sponsored by an agency of the U.S. Government. Neither the U.S. Government nor any agency thereof, not any of their employees, nor any of their contractors, subcontractors or their employees, makes any warranty or representation, express or implied, or assumes any legal liability or responsibility for the accuracy, completeness, or usefulness of any information, apparatus, product, or process disclosed, or represents that its use would not infringe privately own rights. Reference herein to any specific commercial product, process, or service by trade name, trademark, manufacturer, or otherwise, does not necessarily constitute or imply its endorsement, recommendation, or favoring by the U. S. Government or any agency thereof. The views and opinions of authors expressed herein do not necessarily state or reflect those of the U. S. Government or any agency thereof.

\section{REFERENCES}

1. L. M. Barker and R. E. Hollenbach, "Laser interferometer for measuring high velocities of any reflecting surface", J. Appl. Phys. 43, 4669 (1972); L. M. Barker and K. W. Schuler, "Correction to the velocity-perfringe relationship for the VISAR interferometer," J. Appl. Phys. 45, 3692, 1974.

2. D. D. Bloomquist and S. A. Sheffield, "Optically recording interferometer for velocity measurements with sub nanosecond resolution,” J. Appl. Phys. 54, 1717, 1983.

3. R.M. Malone, B.C. Frogget, M.I. Kaufman, P.W. Watts, P.M. Bell, J.R. Celeste, T.L. Lee, "Design of an imaging VISAR diagnostic for the National Ignition Facility (NIF)," SPIE Proc. 5173, 26-37, 2003.

4. Continuum Laser Corporation, Santa Clara, CA.

This work was performed under the auspices of the U.S. Department of Energy by the University of California, Lawrence Livermore National Laboratory under Contract No. W-7405-Eng-48. 